\title{
Bleeding oesophageal varices associated with anabolic steroid use in an athlete
}

\author{
P.J. Winwood, D.A.F. Robertson and Ralph Wright \\ Department of Medicine II, Southampton General Hospital, Southampton SO9 4XY, UK.
}

\begin{abstract}
Summary: A 30 year old bodybuilder who had been taking anabolic steroids for 18 months presented with bleeding oesophageal varices. Serious liver disease secondary to anabolic steroids including peliosis hepatis, nodular hyperplasia and malignant change is well recognized. We report what is, to our knowledge, the first case of bleeding oesophageal varices associated with the use of anabolic steroids.
\end{abstract}

\section{Introduction}

There appears to be widespread use of anabolic steroids amongst athletes ${ }^{1,2}$ leading to increased recognition of adverse effects after long term use, especially gonadal, endocrine and hepatic. Hepatic reactions include cholestasis, peliosis hepatis, regenerative nodular hyperplasia and adenomatous change. ${ }^{3}$ Life threatening complications such as rupture of the liver, malignancy and liver failure are rare but well recognized. ${ }^{3-6}$ Portal hypertension secondary to liver disease resulting from anabolic steroids has previously been implied by the presence of splenomegaly. ${ }^{4}$ We report a bodybuilder who presented with bleeding oesophageal varices having taken long term anabolic steroids.

\section{Case report}

A 30 year old amateur power-lifter, employed as a security consultant, was admitted to Southampton General Hospital having vomited a cupful of fresh blood. Shortly afterwards he vomited a further $1500 \mathrm{ml}$ of fresh blood and clots. He had been taking 3 anabolic steroids for 18 months in doses up to 6 times therapeutic recommendations: stanazolol $15 \mathrm{mg}$, methandienone $15 \mathrm{mg}$ and oxandrolone $7.5 \mathrm{mg}$ daily. He took alcohol rarely and there were no other risk factors for liver disease. Gastroscopy 12 hours after admission revealed 3 grade II oesophageal varices with a bleeding point and overlying blood clot at the gastro-oesophageal junction. No other source of bleeding was identified. On physical examination his musculature was well developed; there were no stigmata of chronic liver disease.

Correspondence: P. Winwood, M.R.C.P.(UK) Accepted: 25 April 1990
He required a blood transfusion of 2 units acutely and his oesophageal varices were treated successfully with sclerotherapy. Liver function tests proved normal except for elevation of the serum aspartate amino transaminase (AST) to 73 IU/1 (normal range; less than 42). Abdominal ultrasound was normal and dynamic isotope liver scan revealed a normal mesenteric fraction $(0.67)$ making portal vein occlusion unlikely. Histology of a needle biopsy of the liver was normal; tests for hepatitis $A$ and $B$ and serum autoantibodies were negative.

The patient stopped taking anabolic steroids, but continued bodybuilding. After 2 weeks the serum AST returned to normal and remained so during the following 6 months. Gastroscopy at this time was normal; there were no oesophageal varices.

\section{Discussion}

In man, continuous long term treatment with $\mathrm{C}-17$ alkylated anabolic steroids (usually longer than 1 year) often causes hepatic disease. ${ }^{3,4}$ Nodular regenerative hyperplasia of hepatocytes and adenomatous change are frequent manifestations. ${ }^{3,4,6}$ Both regenerative hyperplasia and peliosis hepatis secondary to anabolic steroids may be patchy in distribution $^{3-5}$ and liver biopsy as in our patient may show no features of anabolic steroid-induced disease. ${ }^{5}$ Liver function tests are often normal ${ }^{4,5,8}$ or there may be only moderate elevation of the AST. Nodular regenerative hyperplasia may cause portal hypertension ${ }^{7}$ despite a normal liver biopsy. Portal hypertension complicating anabolic steroid-induced hepatic disease has also been suggested by the demonstration of splenomegaly on liver isotope scan in 20 of 33 patients who had been taking long term methyltestosterone. ${ }^{4}$ None of these patients 
had bleeding oesophageal varices and our paper is the first such report.

It has been demonstrated that the Valsalva manoeuvre increases the size and intra-variceal pressure of pre-existing varices. ${ }^{9}$ However, there is no evidence that bodybuilding itself causes oesophageal varices and in this case the varices did not recur despite continued training.

Although the liver biopsy was normal, we believe that this patient's bleeding oesophageal varices resulted from anabolic steroid-induced liver disease, probably regenerative nodular hyperplasia, not seen on liver biopsy because of its patchy distribution. The minimal disturbance of liver function test would be consistent with this diagnosis, although it is also well recognized that bodybuilding may cause elevated serum transaminase levels. ${ }^{10}$ In this patient however the AST

\section{References}

1. Ferner, R.E. \& Rawlins, M.D. Anabolic steroids: the power and the glory? Br Med J 1988, 297: 877-878.

2. Anonymous. Steroids in sports: After four decades, time to return these genies to the bottle? (Editorial). JAMA 1987, 257: 421-427.

3. Ishak, K.G. \& Zimmerman, H.J. Hepatotoxic effects of the anabolic/androgenic steroids. Semin Liver Dis 1987, 7: 230-236.

4. Westaby, D., Ogle, S.J., Paradinas, F.J., Randell, J.B. \& Murray-Lyon, I.M. Liver damage from long-term methyltestosterone. Lancet 1977, ii: 261-263.

5. Bagheri, S.A. \& Boyer, J.L. Peliosis hepatis associated with androgenic-anabolic steroid therapy. Ann Intern Med 1974, 81: $610-618$.

6. Creagh, T.M., Rubin, A. \& Evans, D.J. Hepatic tumours induced by anabolic steroids in an athlete. J Clin Pathol 1988 , 41: $441-443$. remained normal despite resumption of bodybuilding activity. It is also interesting that his varices disappeared so quickly; whilst this may have been due to sclerotherapy, it may be that portal hypertension was a temporary phenomenon occurring whilst the patient was taking anabolic steroids and not due to permanent structural liver disease.

The use of anabolic steroids is widespread amongst bodybuilders. Although neither methandienone nor oxandrolone is available on prescription in the UK, our patient had no difficulty obtaining them. Bleeding oesophageal varices are a possible fatal complication of hepatic disease secondary to anabolic steroids and this case reiterates that both doctors and athletes should be aware of the hazards of long term anabolic steroid use.

7. Stromeyer, F.W. \& Ishak, K.G. Nodular transformation of the liver: a clinicopathologic study of 30 cases. Human Pathol 1981, 12: 60-71.

8. Westaby, D., Portman, B. \& Williams, R. Androgen related primary hepatic tumours in non-Fanconi patients. Cancer 1983, 51: 1947-1952.

9. Palmer, E.D. Effect of Valsalva manoeuvre on portal hypertension in cirrhosis? Am J Med Sci 1954, 227: 661 - 662 .

10. Ross, J.H., Attwood, E.C., Atkin, G.E. \& Villar, R.N. A study on the effects of severe repetitive exercise on serum myoglobin, creatine kinase, transaminases and lactate dehydrogenase. $Q J$ Med 1983, 32: 268-279. 